ESAIM: PROCEEDINGS AND SURVEYS, April 2020, Vol. 67, p. 242-260

Vincent Calvez, Céline Grandmont, Eva Locherbach, Clair Poignard, Magali Ribot, Nicolas Vauchelet Editors

\title{
COMPARISON AND CALIBRATION OF DIFFERENT ELECTROPORATION MODELS. APPLICATION TO RABBIT LIVERS EXPERIMENTS.
}

\author{
Gaspard Jankowiak ${ }^{1}$, Cécile Taing ${ }^{2,3}$, Clair Poignard ${ }^{2,3}$ and Annabelle \\ $\operatorname{COLLIN~}^{4,2,3}$
}

\begin{abstract}
Electroporation is a complex phenomenon that occurs when biological tissues are subjected to electric pulses. The clinical interest for the phenomenon has constantly increased for the last decades. Indeed, electroporation makes it possible to either kill directly the cells in the target region (tumor) or to introduce molecules into living cells. However, one of the main limitation of using electroporation in the clinical routine comes from the technical difficulties raised by such therapies, in particular it is difficult to well determine the treated zone. Numerical modeling of the electric field magnitude could provide a powerful strategy to assess the treatment efficacy: thanks to well-designed models, the computation of the distribution of the electric field is achievable, providing a numerical evaluation of the treatment. The main objective of this work is to go further on the patient-adapted numerical modeling of the electric field magnitude by laying the ground of the possible electroporation models - which will be compared qualitatively - and their calibrations. This will be done in the framework of bioelectrical measurements on rabbit livers that come from the literature.
\end{abstract}

Résumé. L'électroporation est un phénomène complexe qui se produit lorsque des tissus biologiques sont soumis à des impulsions électriques. L'intérêt clinique pour ce phénomène n'a cessé de croître au cours des dernières décennies. En effet, l'électroporation permet soit de tuer directement les cellules dans la région cible (tumeur), soit d'introduire des molécules dans les cellules vivantes. Cependant, l'une des principales limites de l'utilisation de l'électroporation en routine clinique vient des difficultés techniques soulevées par ces thérapies, en particulier la difficulté de bien déterminer la zone traitée. La modélisation numérique de la magnitude du champ électrique pourrait fournir une stratégie puissante pour évaluer l'efficacité du traitement : grâce à des modèles bien conçus, le calcul de la distribution du champ électrique est réalisable, fournissant une évaluation numérique du traitement. L'objectif principal de ce travail est d'aller plus loin dans la modélisation numérique de l'amplitude du champ électrique adaptée au patient en posant les bases des modèles d'électroporation possibles - qui seront comparés qualitativement - et de leurs calibrages. Ceci sera fait dans le cadre de mesures bioélectriques sur des foies de lapins qui proviennent de la littérature.

${ }^{1}$ RICAM - Austrian Academy of Sciences, Postgasse 7-9, 1170, Wien, Austria; e-mail: gaspard@math.janko.fr

2 Univ. Bordeaux, IMB, UMR 5251, F-33400, Talence, France

3 INRIA Bordeaux-Sud-Ouest, F-33400, Talence, France

${ }^{4}$ Bordeaux INP, IMB, UMR 5251, F-33400,Talence, France

(C) EDP Sciences, SMAI 2020

This is an Open Access article distributed under the terms of the Creative Commons Attribution License (http://creativecommons.org/licenses/by/4.0), which permits unrestricted use, distribution, and reproduction in any medium, provided the original work is properly cited. 


\section{INTRODUCTION}

Electroporation (EP) is a complex phenomenon that occurs when biological tissues are subjected to short, high intensity electric pulses. The physical rationale of EP at the cell scale consists of the increase of membrane permeability when the cell is subjected to an electric field strong enough. More precisely, when the cell transmembrane voltage (the difference of the electric potential across the cell membrane) reaches around $1 \mathrm{~V}$, then defects are created in the membrane, which thus becomes permeant to extracellular molecules $[15,16]$.

The clinical interest for the phenomenon has constantly increased for the last decades. Indeed, EP makes it possible to either kill directly the cells in the target region (tumor) by a nonthermal mechanism named irreversible electroporation (IRE) $[5,6,8,11]$, or to introduce non permeant molecules (ions, cytotoxic drugs like bleomycin, DNA plasmids, etc.) into living cells, which is referred to as reversible electroporation $[9,10,17]$. The clinical interests in electroporation-based ablation therapies (EPAs) - either IRE alone or electroporation combined with drug delivery, which is referred to as electrochemotherapy (ECT) - have dramatically increased recently, after publications showing that EPAs provide interesting alternatives to standard non surgical ablative techniques -radiation therapy, radiofrequency ablation (RFA), or cryoablation (CrA) - to treat deep-seated non resectable tumors.

However EPAs are currently mainly limited to cutaneous and subcutaneous tumors. One the main reason of the limitation in the clinical routine use of EPAs comes from the technical difficulties raised by such therapies. Unlike standard ablative techniques which mainly deal with one needle, EPAs need at least two and usually three to four needles (even more for complex tumor shapes): the a priori determination of the treated zone is thus trickier than for standard ablative techniques. Moreover unlike thermal based ablation therapies, as RFA or CrA, for which the monitoring of the temperature in the tumor gives to physician an instantaneous assessment of the treatment, for EPAs the treated region is linked to the amplitude of the electric field, which cannot be monitored instantaneously since the pulse durations are very short (several tens of microseconds per pulse).

Electroporation is tightly linked to the electroquasistatic description of the electric field in the tissue. Therefore numerical modeling of the electric field magnitude provides a powerful strategy to assess the treatment efficacy: thanks to well-designed simulations, the numerical distribution of the electric field is achievable, providing a numerical evaluation of the treatment. However the choice of the model and the patient-dependent calibration, and particularly the patient-dependent organ conductivity, are crucial challenges which are still open.

The aims of the project is to compare the solutions of the different electroporation models found in the literature and to investigate their calibrations for electrostatic descriptions of a tissue from bioelectrical measurements on rabbit livers performed by Sel et al. [19]. After a presentation of 3 different static and 1 dynamical models of electroporation performed in Section 1, Section 2 presents the experimental set-up of Sel et al. and shows how to link the models outputs to the data. After the presentation of numerical schemes used to solve the models, Section 3 proposes a comparison of the solutions in the framework of Sel et al.. Section 4 is devoted to the investigation of parameters estimation from data measurements.

\section{Electroporation models}

In this section, we present three static models and one dynamical model, which provide the electrical description of tissue. To compare the different models, the following simple configuration is considered (see Figure 1). The domain $\tilde{\Omega}$ is a connected, open smooth subset of $\mathbb{R}^{d}, d \in\{2,3\}$, and consists of two non intersecting subsets: $\Omega$, which corresponds to the tissue under consideration (rabbit liver for instance in the experiment of Sel et al.) and the needles, represented by two parallel cylinders $\mathcal{E}^{ \pm}$. The needles are set at the isopotential $\pm g$ respectively. The outer boundary is denoted by $\Gamma_{\text {out }}:=\partial \Omega \backslash \mathcal{E}^{ \pm}$. 


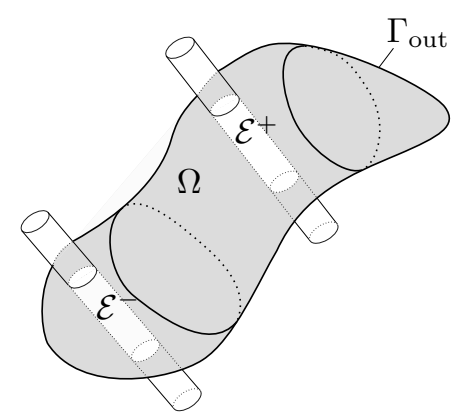

Figure 1. The typical geometrical configuration consists of the tissue domain $\Omega$ in gray, deprived of the two needles $\mathcal{E}^{ \pm}$, in white. The outer boundary $\Gamma_{\text {out }}$ is represented in bold.

\subsection{The standard static model}

The most used model to describe tissue electroporation consists of the phenomenological electrostatic problem. The tissue is described as a conductive medium, whose conductivity tensor $\sigma$ depends on the amplitude of the electric field $-\nabla u$. The model reads then

$$
\begin{aligned}
-\nabla \cdot(\sigma(\|\nabla u\|) \nabla u) & =0, \quad \text { in } \Omega, \\
\left.\partial_{n} u\right|_{\Gamma_{\text {out }}}=0,\left.\quad u\right|_{\mathcal{E}^{ \pm}} & =g^{ \pm},
\end{aligned}
$$

The tissue conductivity consists of a 4 parameters sigmoid function. Typically

$$
\forall \lambda \geq 0, \quad \sigma(\lambda)=\sigma_{0}+\frac{\sigma_{1}-\sigma_{0}}{2}\left(1+\operatorname{erf}\left(\mathrm{k}_{\mathrm{ep}}\left(\lambda-\mathrm{E}_{\mathrm{th}}\right)\right)\right),
$$

where $\sigma_{0}$ is the conductivity of the non electroporated tissue, $\sigma_{1}$ is the tissue conductivity of the fully porated tissue, $\mathrm{E}_{\mathrm{th}}$ is the threshold amplitude for electroporation, and $\mathrm{k}_{\mathrm{ep}}$ is the slope of the nonlinearity. Here, erf is the Gauss error function. The qualitative behaviour of $\sigma$ is depicted in Figure 2.

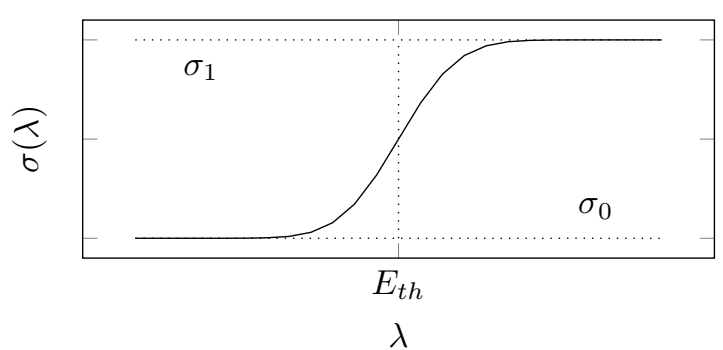

Figure 2. The shape of the conductivity $\sigma$

Remark 1. It should be noted that this choice of $\sigma$ is largely phenomenological, and as shown in [12], the available experimental data does not seem sufficient to characterize the dependence of the conductivity on the electric field.

\subsection{The electric circuit approach of Voyer et al.}

In [20], Voyer et al. proposed a biphasic dynamical model based on the description of an individual cell and surrounding matrix as an electric circuit. The ODEs at the cell level are formally generalized to PDEs at the 
tissue level. It describes the electric potential outside cells $\phi_{e}$ and the electric field inside cells $\mathbf{J}_{c}$. The parameters are the extracellular and intracellular electric conductivities, respectively $\sigma_{e}$ and $\sigma_{c}$. The conductivity of the cell membrane $\sigma_{m}$ depends on time in a way which mimics the effects of poration, i.e. the appearance of holes on the membrane, and permeabilisation, that is the degradation of membrane molecules. Both phenomena increase the conductivity. The resulting system reads

$$
\begin{aligned}
& \nabla \cdot\left(\sigma_{e} \nabla \phi_{e}+\mathbf{J}_{c}\right)=0, \\
& \varepsilon_{m} \partial_{t} \mathbf{J}_{c}+\left(\sigma_{m}\left(t,\left\|\mathbf{E}_{m}\right\|\right)+\sigma_{c}\right) \mathbf{J}_{c}=\sigma_{c} \sigma_{m} \nabla \phi_{e}, \\
& \left.\partial_{n} \phi_{e}\right|_{\Gamma_{\text {out }}}=0,\left.\quad \phi_{e}\right|_{\mathcal{E}^{ \pm}}=g^{ \pm},\left.\quad \mathbf{J}\right|_{\Gamma_{\text {out }}}=0,
\end{aligned}
$$

where $\mathbf{E}_{m}=\nabla \phi_{e}-\sigma_{c}^{-1} \mathbf{J}_{c}$, and

$$
\sigma_{m}\left(t,\left\|\mathbf{E}_{m}\right\|\right)=\sigma_{0}^{m}+\sigma_{1}^{m} X_{1}\left(t,\left\|\mathbf{E}_{m}\right\|\right)+\sigma_{e}^{m} X_{2}\left(t,\left\|\mathbf{E}_{m}\right\|\right) .
$$

The functions $X_{1}$ and $X_{2}$ are the respective degrees of poration and degrees of permeabilisation. They satisfy

$$
\dot{X}_{1}=\frac{\beta_{1}\left(\left\|\mathbf{E}_{m}\right\|\right)-X_{1}}{\tau_{1}}, \text { and } \dot{X}_{2}=\frac{\beta_{2}\left(X_{1}\right)-X_{2}}{\tau_{2}},
$$

where $\beta_{1}$ and $\beta_{2}$ are 2-parameters sigmoid functions of the form

$$
\beta_{i}(x)=\frac{1}{2}\left(1+\operatorname{erf}\left(k_{i}\left(x-x_{i}^{\text {trans }}\right)\right)\right),
$$

where $k_{i}$ is the stiffness of the sigmoid and $x_{i}^{\text {trans }}$ the transition threshold.

A simplified static version of this model allows to compare it with the standard model. Indeed, the static version of this model writes

$$
\begin{aligned}
& \nabla \cdot\left(\sigma_{e} \nabla \phi_{e}+\mathbf{J}_{c}\right)=0 \\
& \left(\sigma_{m}\left(\left\|\mathbf{E}_{m}\right\|\right)+\sigma_{c}\right) \mathbf{J}_{c}=\sigma_{c} \sigma_{m}\left(\left\|\mathbf{E}_{m}\right\|\right) \nabla \phi_{e}, \\
& \left.\partial_{n} \phi_{e}\right|_{\Gamma_{\text {out }}}=0,\left.\quad \phi_{e}\right|_{\mathcal{E}^{ \pm}}=g^{ \pm},\left.\quad \mathbf{J}\right|_{\Gamma_{\text {out }}}=0 .
\end{aligned}
$$

where $\sigma_{m}$ is a sigmoid function similar to (2). Written in $\phi_{e}$ and $\mathbf{E}_{m}$, the equations become

$$
\begin{aligned}
& \nabla \cdot\left(\left(\sigma_{e}+\sigma_{c}\right) \nabla \phi_{e}-\sigma_{c} \mathbf{E}_{m}\right)=0, \quad \text { in } \Omega, \\
& \left(\sigma_{m}\left(\left\|\mathbf{E}_{m}\right\|\right)+\sigma_{c}\right) \mathbf{E}_{m}=\sigma_{c} \nabla \phi_{e}, \quad \text { in } \Omega, \\
& \left.\partial_{n} \phi_{e}\right|_{\Gamma_{\text {out }}}=0,\left.\quad \phi_{e}\right|_{\mathcal{E}^{ \pm}}=g^{ \pm},\left.\quad \mathbf{E}_{m}\right|_{\Gamma_{\text {out }}}=0 .
\end{aligned}
$$

Problem (5) makes it possible to define the equivalent tissue conductivity. Actually, thanks to (5b), the potential $\phi_{e}$ satisfies

$$
\nabla \cdot\left(\left(\sigma_{e}+\frac{\sigma_{c} \sigma_{m}\left(\left\|\mathbf{E}_{m}\right\|\right)}{\sigma_{c}+\sigma_{m}\left(\left\|\mathbf{E}_{m}\right\|\right)}\right) \nabla \phi_{e}\right)=0
$$

The equivalent tissue conductivity can thus be defined as

$$
\sigma_{\mathrm{eq}}\left(\left\|\mathbf{E}_{m}\right\|\right)=\sigma_{e}+\frac{\sigma_{c} \sigma_{m}\left(\left\|\mathbf{E}_{m}\right\|\right)}{\sigma_{c}+\sigma_{m}\left(\left\|\mathbf{E}_{m}\right\|\right)} .
$$


A simplified version of the problem consists in assuming that $\sigma_{m}$ depends on $\left\|\nabla \phi_{e}\right\|$ instead of $\left\|\mathbf{E}_{m}\right\|$. Then the simplified problem reads

$$
\begin{aligned}
& \nabla \cdot\left(\left(\sigma_{e}+\frac{\sigma_{c} \sigma_{m}\left(\left\|\nabla \phi_{e}\right\|\right)}{\sigma_{c}+\sigma_{m}\left(\left\|\nabla \phi_{e}\right\|\right)}\right) \nabla \phi_{e}\right)=0, \quad \text { in } \Omega, \\
& \left.\partial_{n} \phi_{e}\right|_{\Gamma_{\text {out }}}=0,\left.\quad \phi_{e}\right|_{\mathcal{E}^{ \pm}}=g^{ \pm}
\end{aligned}
$$

which is similar to the standard model (1) since the function $\sigma_{e q}$ defined by

$$
\forall \lambda \geq 0, \quad \sigma_{e q}(\lambda)=\sigma_{e}+\frac{\sigma_{c} \sigma_{m}(\lambda)}{\sigma_{c}+\sigma_{m}(\lambda)}
$$

is a sigmoid function.

\subsection{The static bidomain model}

In electrocardiology, the so-called bidomain model has been proven to be the homogenisation limit of the cell scale electric potential. It consists of 2 electric potentials, $u_{e}$ and $v$, which satisfy the following PDE system proposed in [7]:

$$
\begin{aligned}
& \nabla \cdot\left(\left(\sigma_{e}+\sigma_{c}\right) \nabla u_{e}-\sigma_{c} \nabla v\right)=0, \\
& S_{m}\left(\left\|\nabla u_{e}\right\|\right) v-\nabla \cdot\left(\sigma_{c} \nabla v\right)+\nabla \cdot\left(\sigma_{c} \nabla u_{e}\right)=0, \\
& \left.\sigma_{e} \nabla u_{e} \cdot \mathbf{n}\right|_{\Gamma_{\text {out }}}=0,\left.\quad u_{e}\right|_{\mathcal{E}^{ \pm}}=g^{ \pm},\left.\quad \sigma_{c} \nabla v \cdot \mathbf{n}\right|_{\partial \Omega}=\left.\sigma_{c} \nabla u_{e} \cdot \mathbf{n}\right|_{\partial \Omega},
\end{aligned}
$$

where $S_{m}=A_{m} \tilde{S}_{m}$, with $A_{m}\left(\mathrm{~m}^{-1}\right)$ the ratio of membrane area by unit volume and $\tilde{S}_{m}\left(\mathrm{~S} . \mathrm{m}^{-2}\right)$, is the conductance of the cell membranes. This model enables to link rigorously the membrane conductance $S_{m}$ to the electric potential thanks to an homogenization procedure presented in [3,4]. In this sense, it is more physiological than the previous phenomenological models (see Remark 1). To account for the electroporation phenomenon - that is the increase of the membrane conductance - we assume that $\tilde{S}_{m}$ follows a sigmoid function of the norm of the outer electric field $-\nabla u_{e}$

$$
\tilde{S}_{m}\left(\left\|\nabla u_{e}\right\|\right)=\tilde{S}_{m}^{0}+\frac{1}{2}\left(\tilde{S}_{m}^{1}-\tilde{S}_{m}^{0}\right)\left[1+\operatorname{erf}\left(\mathrm{k}_{\mathrm{ep}}\left(\left\|\nabla u_{e}\right\|-\mathrm{E}_{\mathrm{th}}\right)\right)\right] .
$$

Remark 2 (On the nonlinearity). In cardiac electrophysiology, the nonlinearity usually depends on the homogenised transmembrane voltage $u_{e}-u_{c}$. However such a nonlinearity is inconsistent with the electroporation phenomenon. Actually, assuming that $g^{-}=-g^{+}$is constant, the symmetry along the axis $(O y)$ implies that $u_{e}$, $u_{c}$ and the transmembrane voltage vanishes along $(O y)$, which is inconsistent with the fact that electroporation occurs between the needles. For this reason, a nonlinearity depending on $\left\|\nabla u_{e}\right\|$ is chosen, which is relevant with the nonlinearity of the static nonlinear model.

Remark 3 (On the boundary conditions on $v$ ). The Neumann boundary condition satisfied by $v$ states the electric field does not go out of the cell phase, and thus $u_{c}$ given by $u_{c}=u_{e}-v$ satisfies $\left.\partial_{n} u_{c}\right|_{\partial \Omega}=0$.

\section{Data measurements}

The experimental set-up of Sel et al. consists of an ex-vivo cubic piece of rabbit liver in which two needles are inserted (see Figure 3). Square pulses of different amplitudes are applied on the needles and the corresponding intensities are recorded. The data set consists of the measurements of the electric intensity that flows through one needle, say $\mathcal{E}^{+}$for instance. 


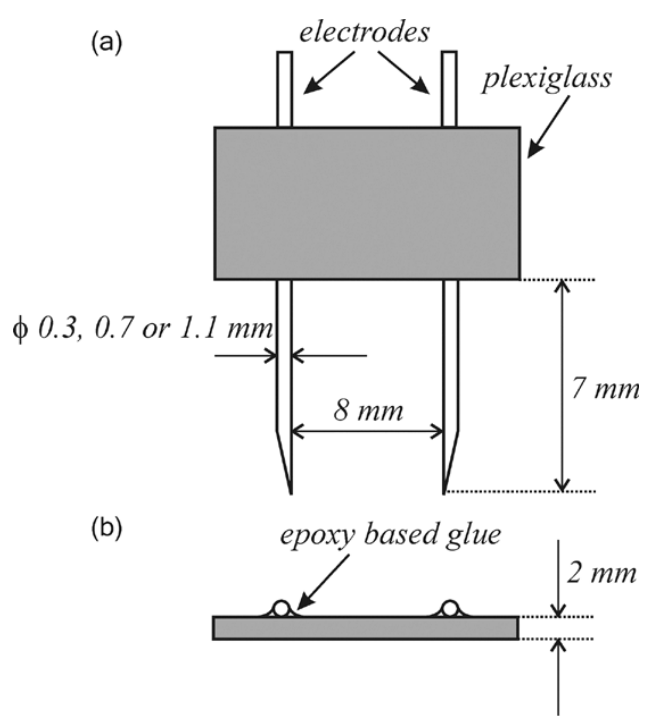

FIGURE 3. Experimental set-up for electroporation measurements as performed by Sel et al. 3 electrodes diameter are considered. The piece of rabbit liver is located below the plexiglass on a thickness of $7 \mathrm{~mm}$ [19].
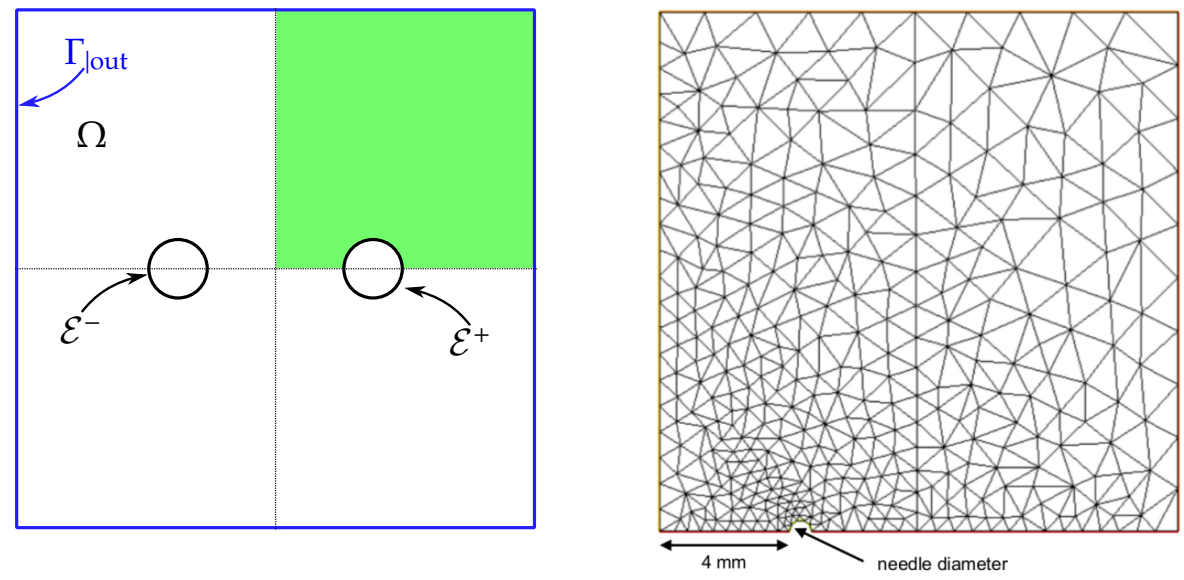

Figure 4. (Left): Symmetrical domain corresponding to the horizontal section of the experimental set-up. (Right): Computational domain restricted to a quadrant. Homogeneous Dirichlet condition is set on the left border, homogeneous Neumann conditions are set on the top, bottom and right borders, non homogeneous Dirichlet condition is set on the needle boundary.

Taking advantage of the symmetries of Figure 4, the computation domain is restricted to a quadrant with homogeneous Neumann conditions on the right, top and bottom borders, homogeneous Dirichlet condition on the left boundary and the non homogeneous Dirichlet condition on the needle. 
The expression of the numerical electric intensity depends on the choice of the models. Denoting by $\ell_{\mathcal{E}^{+}}$the length of the electrode in the perpendicular plane to the 2D simulation plane, one has

$$
\begin{aligned}
& \mathrm{I}_{\mathcal{E}}^{1} \quad=\ell_{\mathcal{E}^{+}} \int_{\mathcal{E}^{+}} \sigma(\|\nabla V\|) \nabla V \cdot \mathbf{n} d s, \quad \text { where } V \text { is the solution to (1), } \\
& \mathrm{I}_{\mathcal{E}}^{2} \quad=\ell_{\mathcal{E}^{+}} \int_{\mathcal{E}^{+}} \sigma_{\mathrm{eq}}\left(\left\|\mathbf{E}_{m}\right\|\right) \nabla \phi_{e} \cdot \mathbf{n} d s, \quad \text { where }\left(\phi_{e}, \mathbf{E}_{m}\right) \text { is the solution to (5), or to (3) } \\
& \mathrm{I}_{\mathcal{E}}^{2, s i m p} \quad=\ell_{\mathcal{E}^{+}} \int_{\mathcal{E}^{+}} \sigma_{\text {eq }}\left(\left\|\nabla \phi_{e}\right\|\right) \nabla \phi_{e} \cdot \mathbf{n} d s, \quad \text { where } \phi_{e} \text { is the solution to (7), } \\
& \mathrm{I}_{\mathcal{E}}^{3} \quad=\ell_{\mathcal{E}^{+}} \int_{\mathcal{E}^{+}} \sigma_{e} \nabla u_{e} \cdot \mathbf{n} d s, \quad \text { where }\left(u_{e}, v\right) \text { is the solution to (8). }
\end{aligned}
$$

The above definition of the electrical intensities of the models involve the gradient of the solution along the needle. This is numerically unstable since the electric field is the most intense nearby the electrodes, and thus numerical instabilities may appear. To smoothen this behavior, it is possible to introduce a specific function $w$ such that the surface integral is replaced by a volume integral. More precisely, let $w$ be defined by

$$
\begin{aligned}
& -\Delta w=0, \quad \text { in } \Omega, \\
& \left.w\right|_{\mathcal{E}^{ \pm}}= \pm 1,\left.\quad \nabla w \cdot \mathbf{n}\right|_{\Gamma_{\text {out }}}=0 .
\end{aligned}
$$

Then, thanks to appropriate integration by parts one has

$$
\begin{aligned}
\mathrm{I}_{\mathcal{E}}^{1} & =\frac{\ell_{\mathcal{E}}}{2} \int_{\Omega} \sigma(\|\nabla V\|) \nabla V \cdot \nabla w d x, & & \text { where } V \text { is the solution to (1), } \\
\mathrm{I}_{\mathcal{E}}^{2} & =\frac{\ell_{\mathcal{E}}^{+}}{2} \int_{\Omega} \sigma_{\mathrm{eq}}\left(\left\|\mathbf{E}_{m}\right\|\right) \nabla \phi_{e} \cdot \nabla w d x, & & \text { where }\left(\phi_{e}, \mathbf{E}_{m}\right) \text { is the solution to (5), } \\
\mathrm{I}_{\mathcal{E}}^{2, \text { simp }} & =\frac{\ell_{\mathcal{E}}}{2} \int_{\Omega} \sigma_{\mathrm{eq}}\left(\left\|\nabla \phi_{e}\right\|\right) \nabla \phi_{e} \cdot \nabla w d x, & & \text { where } \phi_{e} \text { is the solution to (7), or to (3) } \\
\mathrm{I}_{\mathcal{E}}^{3} & =\frac{\ell_{\mathcal{E}}^{+}}{2} \int_{\Omega}\left(\left(\sigma_{e}+\sigma_{c}\right) \nabla u_{e}-\sigma_{c} \nabla v\right) \cdot \nabla w d x, & & \text { where }\left(u_{e}, v\right) \text { is the solution to (8). }
\end{aligned}
$$

\section{Numerical Discretization AND Qualitative COMPARISON BETWEen The ModelS}

\subsection{Discretization and numerical approximation}

In this section, we present the discretizations that have been used to solve the different models. All these strategies have been implemented using the Python finite element library FEniCS [1].

\subsubsection{Static models: illustration on the standard static model}

We want to solve (1) which we recall here:

$$
\begin{aligned}
-\nabla \cdot(\sigma(\|\nabla u\|) \nabla u) & =0, \\
\left.\partial_{n} u\right|_{\Gamma_{\text {out }}}=0,\left.\quad u\right|_{\mathcal{E}^{ \pm}} & =g^{ \pm},
\end{aligned}
$$

which is a non-linear problem since $\sigma$ depends on $\nabla u$. In Breton et al. [2] a modified fixed point has been presented and the numerical convergence of this scheme has been shown. In this work, we compare two other strategies. The first one consists in using iteration schemes coming from the time-discretization of the following non-linear evolution equation

$$
\partial_{s} u-\nabla \cdot(\sigma(\|\nabla u\|) \nabla u)=0 \quad \text { in } \Omega,
$$


with the same boundary conditions as above, and where $s$ acts as the pseudo time variable. We follow the same strategy for the static version of the biphasic model and for the bidomain model. Depending on the eigenvalue of the linearised problem, one can expect that $u$ behaves asymptotically as the static solution. We explicit the strategy (which can be easily extended to the other static models) for the standard model. First, we consider the decomposition $u=v+u_{\text {lin }}$, where $u_{\text {lin }}$ solves the linear static problem associated to (1), that is the Laplace equation on $\Omega$ with boundary conditions (1b). Thus, we obtain the following equation on $v$

$$
\partial_{s} v-\nabla \cdot\left(\sigma\left(\left\|\nabla\left(v+u_{\operatorname{lin}}\right)\right\|\right) \nabla\left(v+u_{\operatorname{lin}}\right)\right)=0,
$$

with homogeneous Dirichlet boundary conditions, since $u_{\text {lin }}$ carries the boundary conditions of (1). By discretizing the $s$ derivative by finite differences with step $\Delta s$, multiplying this equation by a test function $\varphi \in C_{c}^{\infty}(\Omega)$ and integrating over $\Omega$, we get

$$
\begin{aligned}
\int_{\Omega} \frac{v_{n+1}-v_{n}}{\Delta s} \varphi & +\int_{\Omega} \sigma\left(\left\|\nabla\left(v_{n}+u_{\text {lin }}\right)\right\|\right) \nabla v_{n+1} \cdot \nabla \varphi+\int_{\Omega} \sigma\left(\left\|\nabla\left(v_{n}+u_{\text {lin }}\right)\right\|\right) \nabla u_{\text {lin }} \cdot \nabla \varphi \\
& -\int_{\mathcal{E}^{ \pm} \cup \Gamma_{\text {out }}} \sigma\left(\left\|\nabla\left(v_{n}+u_{\text {lin }}\right)\right\|\right) \nabla v_{n+1} \cdot \mathbf{n} \varphi=0 .
\end{aligned}
$$

The term on $\Gamma_{\text {out }}$ vanishes because of the homogeneous Neumann boundary conditions, and we can restrict to test functions with zero trace on $\mathcal{E}^{ \pm}$by integrating the Dirichlet data in the function space, see below. Hence, the variational problem to solve reads $A\left(v_{n+1}, \varphi\right)=L(\varphi)$ where

$$
\begin{aligned}
& A\left(v_{n+1}, \varphi\right):=\int_{\Omega} v_{n+1} \varphi+\Delta s \int_{\Omega} \sigma\left(\left\|\nabla\left(v_{n}+u_{\operatorname{lin}}\right)\right\|\right) \nabla v_{n+1} \cdot \nabla \varphi, \\
& L(\varphi):=\int_{\Omega} v_{n} \varphi-\Delta s \int_{\Omega} \sigma\left(\left\|\nabla\left(v_{n}+u_{\operatorname{lin}}\right)\right\|\right) \nabla u_{\text {lin }} \cdot \nabla \varphi .
\end{aligned}
$$

This iterative method is initialized by taking $v_{0}=u_{\operatorname{lin}}$. We then proceed by approximating $v, u_{\operatorname{lin}}$ using $\mathbb{P}_{2}$ finite elements, i.e. we have $v^{h}, u_{\operatorname{lin}}^{h} \in V^{h}$ with

$$
V^{h}=\left\{w \in C^{0}(\Omega):\left.w\right|_{T} \in \mathbb{P}^{2} \forall T \in \mathcal{T}_{h}\right\}
$$

where $\mathbb{P}^{2}$ is the space of polynomials in two variables, of degree at most 2 and $\mathcal{T}_{h}$ is a triangulation of $\Omega$. We also introduce

$$
V_{\psi}^{h}:=\left\{w \in V^{h}:\left.w\right|_{\mathcal{E}^{ \pm}}=\psi\right\} .
$$

We are left with the problem of finding $v_{n+1}^{h} \in V_{g^{ \pm}}^{h}$ such that

$$
A\left(v_{n+1}^{h}, \varphi^{h}\right)=L\left(\varphi^{h}\right), \quad \forall \varphi^{h} \in V_{0}^{h},
$$

for $n>0$, with $v_{0}^{h}=u_{\text {lin }}^{h}$. This problem is well-posed according to standard finite element analysis arguments.

The second approach consists in using Newton's method directly on the finite element system. Consider the problem consisting in finding $u^{h} \in V_{g^{ \pm}}^{h}$ such that

$$
\mathcal{A}\left(u^{h}, \varphi^{h}\right)=0, \quad \forall \varphi^{h} \in V_{0}^{h},
$$

where $\mathcal{A}$ is given by

$$
\mathcal{A}\left(u^{h}, \varphi^{h}\right)=\int_{\Omega} \sigma\left(\left\|\nabla u^{h}\right\|\right) \nabla u^{h} \cdot \nabla \varphi^{h}
$$


which is the discretized weak formulation of (1). Assuming that some $u_{0}^{h}$ satisfying the boundary conditions is known, one can try to solve this system of non linear equations using the Newton's method. Such a $u_{0}^{h}$ is given by solving Laplace's equation on $\Omega$ with boundary conditions (1b). In FEniCS, the computation of the corresponding Jacobian can be performed automatically. For the models we consider, this approach is faster and more stable compared to the evolution-based iterative method presented above, although it requires a good guess for initialization. In practice, such a good guess can be found by starting from the solution to the linear problem and increasing gradually the value of $k_{e p}$ until the desired value is reached. The Newton's approach is also simpler, in the sense that is does not require a choice of a step size ( $\Delta s$ in the evolution approach).

A qualitative comparison of the two methods can be seen in Figure 5. $\sigma(\|\nabla u\|)$ is projected on piecewise constant discontinuous elements. On the right of the domain, the profile given by the Newton's method is noticeably smoother.
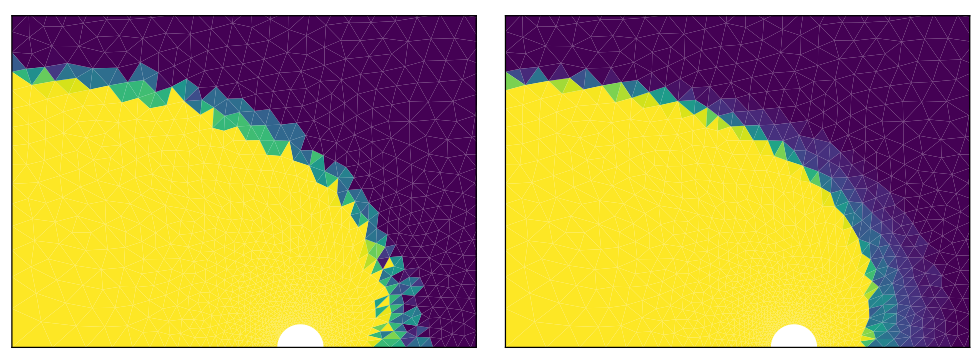

Figure 5. Comparison of $\sigma(\|\nabla u\|)$ for typical solutions of 1 given by the evolution-based iterative method (left) and the Newton's approach (right) for the standard model.

\subsubsection{The dynamical biphase model}

Concerning the dynamical biphase model, we use an iterative time discretization method. We denote the time step by $\Delta t$, so that $t^{n}=n \Delta t$. At the $n^{t h}$ step, we compute the membrane electric field, and its amplitude

$$
\mathbf{E}_{m}^{n}=\nabla u_{e}^{n}-\frac{1}{\sigma_{c}} \mathbf{J}_{c}^{n}, \quad A_{m}^{n}=d_{c}\left|\mathbf{E}_{m}^{n}\right|,
$$

where $d_{c}$ is the mean of the cell diameters. From there, we solve the differential equations for the degrees of poration and permeabilization $X_{1}$ and $X_{2}$ using an explicit Euler scheme

$$
\begin{aligned}
& X_{1}^{n}=X_{1}^{n-1}+\frac{\Delta t}{\tau_{1}}\left(\beta_{1}\left(A_{m}^{n}\right)-X_{1}^{n-1}\right), \\
& X_{2}^{n}=X_{2}^{n-1}+\frac{\Delta t}{\tau_{2}}\left(\beta_{1}\left(X_{1}^{n-1}\right)-X_{2}^{n-1}\right) .
\end{aligned}
$$

Then, we compute the membrane conductivity

$$
\sigma_{m}^{n}=\sigma_{0}^{m}+\sigma_{1} X_{1}^{n}+\sigma_{2} X_{2}^{n} .
$$

Similarly to the static models, we multiply (3) by the test functions $\varphi_{1} \in C^{\infty}(\Omega)$ and $\varphi_{2} \in C_{0}^{\infty}(\Omega)^{2}$ respectively and integrate by parts to obtain

$$
\begin{aligned}
-\sigma_{e} \int_{\Omega} \nabla \phi_{e}^{n+1} \cdot \nabla \varphi_{1}-\sigma_{c} \int_{\Omega} \mathbf{J}_{c}^{n+1} \cdot \nabla \varphi_{1}+\sigma_{e} \int_{\mathcal{E}^{ \pm} \cup \Gamma_{\text {out }}} \nabla \phi_{e}^{n+1} \cdot \mathbf{n} \varphi_{1}+\sigma_{c} \int_{\mathcal{E}^{ \pm} \cup \Gamma_{\text {out }}} \mathbf{J}_{c}^{n+1} \cdot \mathbf{n} \varphi_{1}=0 \\
\varepsilon_{m} \int_{\Omega}\left(\mathbf{J}_{c}^{n+1}-\mathbf{J}_{c}^{n}\right) \cdot \varphi_{2}+\Delta t\left(\int_{\Omega}\left(\sigma_{m}^{n}+\sigma_{c}\right) \mathbf{J}_{c}^{n+1} \cdot \varphi_{2}-\int_{\Omega} \sigma_{m}^{n} \sigma_{c} \nabla \phi_{e}^{n+1} \cdot \varphi_{2}\right)=0 .
\end{aligned}
$$


We discretize again on $\mathbb{P}^{2}$ finite elements and integrate the Dirichlet boundary conditions in the finite element space. We then have to find $\phi_{e}^{n+1, h} \in V_{g^{ \pm}}^{h}, \mathbf{J}_{c}^{n+1} \in\left(V_{0}^{h}\right)^{2}$ such that

$$
\mathfrak{A}\left(\Phi^{n+1, h}, \Psi^{h}\right)=\mathfrak{L}_{n}\left(\Psi^{h}\right),
$$

where $\Phi^{h}=\left(\phi_{e}^{n+1, h}, \mathbf{J}_{c}^{n+1, h}\right)$ and $\Psi^{h}=\left(\varphi_{1}^{h}, \varphi_{2}^{h}\right) \in V_{0}^{h} \times\left(V_{0}^{h}\right)^{2}$. The functionals $\mathfrak{A}$ and $\mathfrak{L}$ are given by:

$$
\begin{aligned}
\mathfrak{A}\left(\Phi^{n+1, h}, \Psi^{h}\right):= & -\sigma_{e} \int_{\Omega} \nabla \phi_{e}^{n+1, h} \cdot \nabla \varphi_{1}^{h}-\sigma_{c} \int_{\Omega} \nabla \mathbf{J}_{c}^{n+1, h} \cdot \nabla \varphi_{1}^{h} \\
& +\epsilon_{m} \int_{\Omega} \mathbf{J}_{c}^{n+1, h} \cdot \varphi_{2}^{h}+\Delta t\left(\int_{\Omega}\left(\sigma_{m}^{n}+\sigma_{c}\right) \mathbf{J}_{c}^{n+1, h} \cdot \varphi_{2}^{h}-\int_{\Omega} \sigma_{m}^{n} \sigma_{c} \nabla \phi_{e}^{n+1, h} \cdot \varphi_{2}^{h}\right), \\
\mathfrak{L}_{n}(\varphi):= & \epsilon_{m} \int_{\Omega} \mathbf{J}_{c}^{n} \cdot \varphi_{2}^{h} .
\end{aligned}
$$

\subsection{Qualitative comparison between the models}

In this section we show the characteristic solution of all three models presented in Section 1. We consider an electrode diameter of $0.7 \mathrm{~mm}$, a voltage of $800 \mathrm{~V}$. Results for the standard, the bidomain and the dynamical biphase models are illustrated in Figures 6, 7 and 8 respectively. In all cases, the contour lines of $u$ and $u_{e}$ look similar.
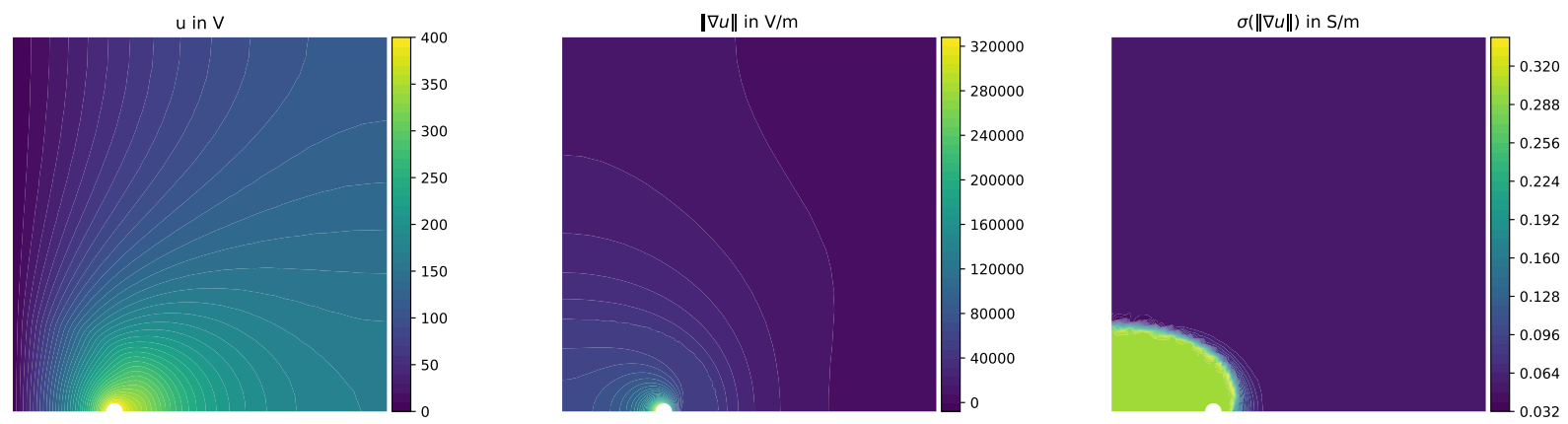

FiguRE 6. Solution of the static standard model, using parameters $E_{t h}=5.75 \times 10^{4} \mathrm{~V} / \mathrm{m}$, $k_{e p}=10^{-3} V^{-1}, \sigma_{0}=0.065 \mathrm{~S} / \mathrm{m}$ and $\sigma_{1}=0.1483 \mathrm{~S} / \mathrm{m}$.

\section{Estimation STRATEGy: ILLUSTRATIONS ON THE STANDARD STATIC MODEL}

As we have seen in the previous section, the solutions of the different models are realistic and can be qualitatively compared. They allow to represent the electroporation phenomena. The aim of this section is to investigate numerical calibration strategies of these models. We will focus for this work on the standard static model.

First, the estimation strategy is introduced. Then, it is illustrated with synthetic data and finally, it is applied on real data [19] presented in Section 2. 

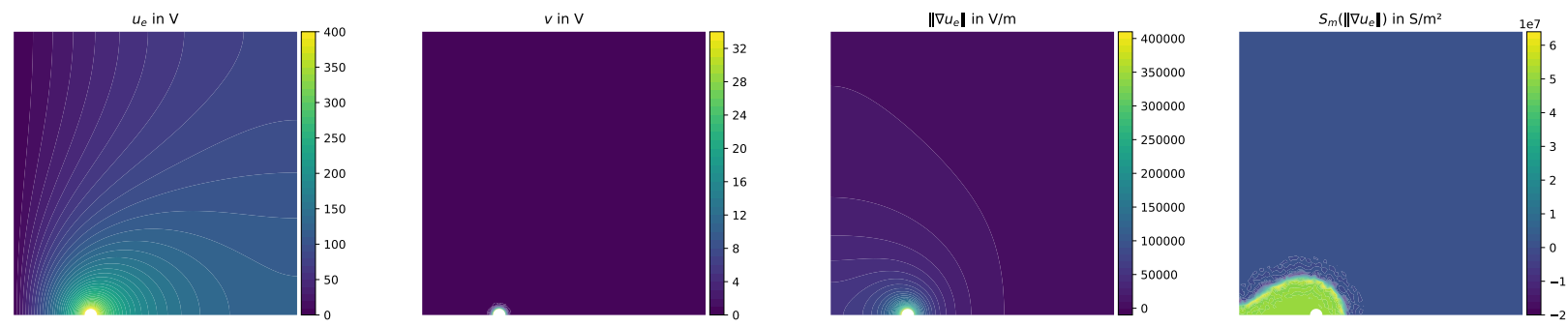

FiguRE 7. Solution of the static bidomain model, using the parameters of [7, Table 3.2]
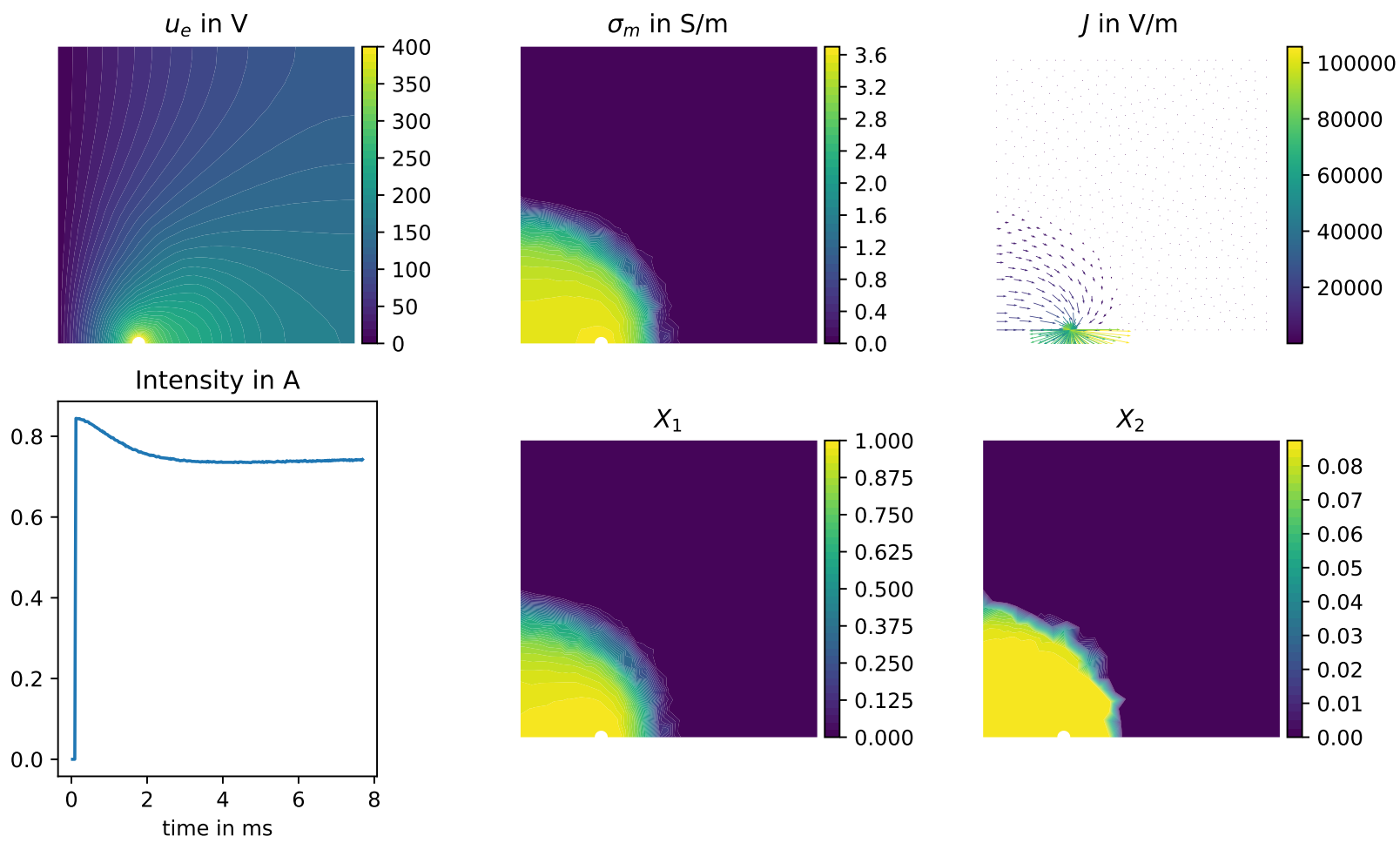

FiguRE 8. Solution to the biphase model at around $8 \mathrm{~ms}$, using the parameters of [20, Table 1 ], with a $800 \mathrm{~V}$ voltage step. It is easy to see that $\sigma_{m}$ is mainly driven by the value of $X_{1}$, i.e. the poration process. At the lower left is the evolution of the current intensity flowing through the electrodes, the spike qualitatively matches experiments, see [20].

\subsection{Parameters estimation algorithm}

We denote by $\theta \in \Theta$ the vector which concatenates all the parameters which have to be estimated. We denote by $y \in \mathcal{Y}$ the solution and by $z \in \mathcal{Z}$ the observations. We denote by $\|\cdot\|_{\Theta,\left(P_{\diamond}\right)^{-1}}$ and by $\|\cdot\|_{\mathcal{Z}, R}$ the norms 
defined by

$$
\|\cdot\|_{\Theta,\left(P_{\diamond}\right)^{-1}}^{2}=\left\langle\cdot,\left(P_{\diamond}\right)^{-1} \cdot\right\rangle \text { and }\|\cdot\|_{\mathcal{Z}, R}^{2}=\langle\cdot, R \cdot\rangle .
$$

The static problem can be rewritten as $\mathcal{A}(y, \theta)=0$, where $\mathcal{A}$ corresponds to the model operator. We denote by $\mathcal{C}$ the observation operator which relies $y$ to $z$

$$
z=\mathcal{C}(y)
$$

and we denote by $\mathcal{O}$ the following operator

$$
\mathcal{O}: \theta \mapsto y \text {, such that } \mathcal{A}(y, \theta)=0 .
$$

Our objective is to inverse the operator $\Psi=\mathcal{C} \circ \mathcal{O}$. In order to do that, the criterium that we want to minimize is given by

$$
\mathcal{J}(\theta)=\frac{1}{2}\left\|\theta-\theta_{\diamond}\right\|_{\Theta,\left(P_{\diamond}\right)^{-1}}^{2}+\frac{1}{2}\|z-\Psi(\theta)\|_{\mathcal{Z}, R}^{2}
$$

where $\theta_{\diamond}$ corresponds to an a priori value of $\theta$. This criterium corresponds to a likelihood functional where all disturbances are assumed to be Gaussian

$$
\theta \sim \mathcal{N}\left(\theta_{\diamond}, P_{\diamond}^{1 / 2}\right) \text { and } z \sim \mathcal{N}\left(\Psi(\theta), R^{-1 / 2}\right)
$$

If we want to use $N_{z}$ measurements denoted by $\left(z_{k}\right)_{1 \leq k \leq N_{z}}$ associated to $\left(R_{k}\right)_{1 \leq k \leq N_{z}}$, we have to consider the following criterium

$$
\mathcal{J}(\theta)=\frac{1}{2}\left\|\theta-\theta_{\diamond}\right\|_{\Theta,\left(P_{\diamond}\right)^{-1}}^{2}+\frac{1}{2} \sum_{k=1}^{N_{z}}\left\|z_{k}-\Psi(\theta)\right\|_{\mathcal{Z}, R_{k}}^{2}
$$

which can be rewritten as

$$
\mathcal{J}(\theta)=\frac{1}{2}\left\|\theta-\theta_{\diamond}\right\|_{\Theta,\left(P_{\diamond}\right)^{-1}}^{2}+\frac{1}{2} \sum_{k=1}^{N_{z}}\left\|^{b} z-{ }^{b} \Psi(\theta)\right\|_{b}^{2} \mathcal{Z},{ }^{b} R
$$

with

$$
{ }^{\mathrm{b}} z=\left(\begin{array}{c}
z_{1} \\
\vdots \\
z_{N_{z}}
\end{array}\right),{ }^{\mathrm{b}} \Psi=\left(\begin{array}{c}
\Psi \\
\vdots \\
\Psi
\end{array}\right),{ }^{\mathrm{b}} \mathcal{Z}=\mathcal{Z} \times \cdots \times \mathcal{Z} \text { and }{ }^{\mathrm{b}} R=\left(\begin{array}{ccc}
R_{1} & & \\
& \ddots & \\
& & R_{N_{z}}
\end{array}\right)
$$

It exists many methods to solve this kind of problem as for example as a non-exhaustive list: gradient descent based methods ; stochastic strategies as for example Monte-Carlo based strategies or expectation-maximization algorithm. In this work, we decided to use a stochastic algorithm based on an unscented transform [14]. Let $\left(\omega^{i}\right)_{1 \leq i \leq p}$ and $\left(e^{(i)}\right)_{1 \leq i \leq p}$ be some weight parameters and directions in $\Theta$ such that

$$
\sum_{1 \leq i \leq p} \omega^{i} e^{(i)}\left(e^{(i)}\right)^{T}=\frac{1}{\rho}
$$

This is called a set of sigma points and many different sets of sigma points have been proposed in the literature. In this work, we will consider the simplex sigma points, which allow to consider only $\operatorname{dim}_{\Theta}+1$ points [13]. The 
algorithm reads as follows:

- Initialisation: $\hat{\theta}_{0}=\theta_{\diamond}$ and $P_{0}=P_{\diamond}$.

- Generation of $p$ particles of mean $\hat{\theta}_{k}$ and covariance $P_{k}$ :

$$
\theta^{(i)}=\hat{\theta}_{k}+\sqrt{\rho P_{k}} e^{(i)}, \quad 1 \leq i \leq p .
$$

- Computation of the observations generated by the solution of the system for each particle:

$$
{ }^{\mathrm{b}} Z^{i}={ }^{\mathrm{b}} \Psi\left(\theta^{(i)}\right) .
$$

- Computation of the mean and of the covariance of ${ }^{b} Z^{i}$ :

$$
{ }^{b} Z^{*}=\mathbb{E}\left({ }^{b} Z^{i}\right) \stackrel{\text { def }}{=} \sum \omega^{i b} Z^{i} \text { and } P^{z}=\operatorname{Cov}\left({ }^{b} Z^{i}\right) \stackrel{\text { def }}{=} \sum \omega^{i}\left({ }^{b} Z^{i}-{ }^{b} Z^{*}\right)\left({ }^{b} Z^{i}-{ }^{b} Z^{*}\right)^{T} .
$$

- Computation of the covariance between $\theta^{(i)}$ and $J\left(\theta^{(i)}\right)$ (sensitivity of the observations to the parameters):

$$
P^{\theta z}=\operatorname{Cov}\left(\theta^{(i)}, J\left(\theta^{(i)}\right)\right) \stackrel{\text { def }}{=} \sum \omega^{i}\left(\theta^{(i)}-\hat{\theta}_{k}\right)\left({ }^{b} Z^{i}-{ }^{b} Z^{*}\right)^{T} .
$$

- Computation of the new value of the parameters and of their covariances

$$
\begin{aligned}
& \hat{\theta}_{k+1}=\hat{\theta}_{k}+P^{\theta z}\left(P^{z}+R^{-1}\right)^{-1}\left({ }^{b} z-{ }^{b} Z^{*}\right), \\
& P_{k+1}=P_{k}-P^{\theta z}\left(P^{z}+R^{-1}\right)^{-1}\left(P^{\theta z}\right)^{T} .
\end{aligned}
$$

\subsection{Estimations on synthetic data}

As said previously, we focus on the standard static model in this section. The parameters are then the parameters of the sigmoid function: $E_{t h}, \sigma_{0}, \sigma_{1}$ and $k_{e p}$. We want to validate on synthetic data the estimation strategy. To do that, we consider a framework close from the real data. Following [19], we build synthetic data with intensity measurements for 5 different voltages and 3 electrode sizes (the same of the article). The corresponding observation space has then dimension $N_{z}=15$. The synthetic data are obtained using $E_{t h}=$ $5.75 \times 10^{4} \mathrm{~V} / \mathrm{m}, \sigma_{0}=0.065 \mathrm{~S} / \mathrm{m}, \sigma_{1}=0.1483 \mathrm{~S} / \mathrm{m}$ and $k_{e p}=10^{-3}$. The parameters that we want to estimate are all positive which will be constrained by a log-transformation during the estimation procedure. We assume that

$$
\begin{aligned}
& \log E_{t h} \sim \mathcal{N}\left(\log \left(4.5 \times 10^{4}\right), \sqrt{0.2}\right), \log \sigma_{0} \sim \mathcal{N}(\log (0.2), \sqrt{0.2}) \\
& \log \sigma_{1} \sim \mathcal{N}(\log (0.09), \sqrt{0.2}) \text { and } \log k_{e p} \sim \mathcal{N}\left(\log \left(10^{-4}\right), \sqrt{0.2}\right)
\end{aligned}
$$

Concerning the uncertainties quantification of the observations, we assume that ${ }^{b} R^{-1 / 2}=0.025 \operatorname{Id}_{N_{z}, N_{z}}$ which corresponds to a noise standard deviation of approximately $20 \%: R^{-1 / 2} \approx 20 \% \frac{1}{N_{z}} \sum_{k=1}^{N_{z}}\left|z_{k}\right| \operatorname{Id}_{N_{z}, N_{z}} \approx 0.025$. First of all, we start by an individual estimation of the parameters and the synthetic data are not noisy. Table 1 presents the results (with 2 digits of precision). The algorithm stops when the convergence is reached.

As a conclusion, it is possible to estimate three parameters: $E_{t h}, \sigma_{0}$ and $\sigma_{1}$. The sensitivity of $\sigma_{0}$ is weaker and more iterations are needed (compared to $E_{t h}$ and $\sigma_{1}$ ). Then, $k_{e p}$ is not identifiable: there is no sensitivity associated to this parameter. As $k_{e p}$ is not identifiable, we fix it at its true value and we make a coupled estimation of the three other parameters, see the third column of Table 2. We consider also a case where only one size of electrodes is considered. The results are very encouraging and show that it is possible to estimate the three parameters together. The results are slightly better when three electrodes are considered. Finally, we add a gaussian noise of standard deviation $\sigma_{n}=0.005,0.01$ (resp. $5 \%$ and 10\%) on the synthetic data to see the sensitivity of the estimation to measurement noise. In the case of $\sigma_{n}=0.01$, we also try to estimate using 


\begin{tabular}{cccc}
\hline Parameter & Real value & Estimated value & Number of iterations \\
\hline$E_{t h}$ & 10.96 & 10.95 & 13 \\
$\sigma_{0}$ & -2.73 & -2.74 & 20 \\
$\sigma_{1}$ & -1.91 & -1.91 & 3 \\
$k_{e p}$ & -6.91 & -9.20 & $\infty$ \\
\hline
\end{tabular}

TABLE 1. Individual estimation of the parameters ( 2 digits of precision). The true and estimated values are given after the log-transformation.

\begin{tabular}{cccc}
\hline Parameter & Real value & Estim. & Estim., 1 elec. \\
\hline$E_{t h}$ & 10.96 & 10.95 & 10.95 \\
$\sigma_{0}$ & -2.73 & -2.76 & -2.77 \\
$\sigma_{1}$ & -1.91 & -1.90 & -1.88 \\
\hline
\end{tabular}

TABLE 2. Coupled estimation of $E_{t h}, \sigma_{0}$ and $\sigma_{1}$ (2 digits of precision). The true and estimated values are given after the log-transformation. The third (resp. fourth) column corresponds to the case where the 3 (resp. 1) electrode(s) are (resp. is) considered.

\begin{tabular}{ccccc}
\hline Parameter & Real value & Estim. $\sigma_{n}=0.005$ & Estim. $\sigma_{n}=0.01$ & Estim. $\sigma_{n}=0.01,1$ elec. \\
\hline$E_{t h}$ & 10.96 & 10.94 & 10.83 & 10.86 \\
$\sigma_{0}$ & -2.73 & -2.75 & -2.72 & -2.59 \\
$\sigma_{1}$ & -1.91 & -1.91 & -1.94 & -1.98 \\
\hline
\end{tabular}

TABLE 3. Coupled estimation of $E_{t h}, \sigma_{0}$ and $\sigma_{1}$ (2 digits of precision). The true and estimated values are given after the log-transformation. The third (resp. fourth) column corresponds to $\sigma_{n}=0.005$ (resp. $\sigma_{n}=0.01$ ). The last column is for $\sigma_{n}=0.01$ and only one electrode is considered.

only one size of electrodes. The results are given in Table 3. As expected, the more the noise on the data is important, the more it is difficult to well estimate the parameters but the results are still encouraging. More precisely, with noisy data, it is crucial to consider measurements performed with different size of electrodes.

\subsection{Estimations on the data set of Sel et al.}

We now turn to the problem of parameter estimation for real data.

\subsubsection{Dataset}

The dataset used is the one provided by [19], the corresponding experimental setup is recalled in Figure 3 . It provides intensity measurements for 5 different voltages and 3 electrode sizes, which are collected in Table 4 . As for the synthetic data, the corresponding observation space has then dimension $N_{z}=15$. 


\begin{tabular}{cccccc}
\hline Parameter & Initial value & Estim. 0.3mm & Estim. 0.7mm & Estim 1.1mm & Estim all 3 \\
\hline$E_{t h}$ & 10.96 & 10.9 & 9.59 & 9.60 & 9.75 \\
$\sigma_{0}$ & -2.73 & -1.88 & -5.78 & -4.23 & -9.06 \\
$\sigma_{1}$ & -1.909 & -0.37 & -0.58 & -0.77 & -0.65 \\
\hline
\end{tabular}

TABLE 5. Initial values and results of the 4 estimations, one for each electrode diameter, and one with all diameters combined.

\begin{tabular}{cccccc}
\hline Electrode diameter & \multicolumn{5}{c}{ Applied voltage } \\
\hline & $200 \mathrm{~V}$ & $400 \mathrm{~V}$ & $600 \mathrm{~V}$ & $800 \mathrm{~V}$ & $1000 \mathrm{~V}$ \\
\cline { 2 - 6 } $0.3 \mathrm{~mm}$ & 0.104 & 0.275 & 0.571 & 0.877 & 1.260 \\
$0.7 \mathrm{~mm}$ & 0.170 & 0.412 & 0.850 & 1.389 & 1.663 \\
$1.1 \mathrm{~mm}$ & 0.168 & 0.437 & 0.776 & 1.315 & 1.533 \\
\hline
\end{tabular}

TABLE 4. Intensity (A) of the current flowing through the electrodes measured by $[19]$

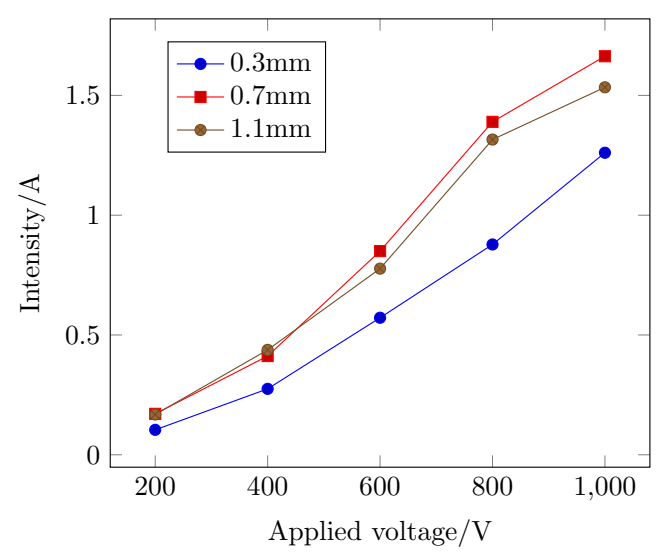

\subsubsection{Estimation and validation}

As was made clear in Section 4.2, the sensivity on $k_{e p}$ in the case of a 4-parameter sigmoid is very low. Trying to estimate this parameter would most likely result in an unreliable value. To avoid having to choose an arbitrary value for $k_{e p}$, we consider a 3-parameter sigmoid instead, of the form

$$
\sigma(\|\nabla V\|)=\sigma_{0}+\left(\sigma_{1}-\sigma_{0}\right) \exp \left(\frac{E_{t h}^{2}}{\|\nabla V\|^{2}}\right) \text {. }
$$

The remaining three parameters, $E_{t h}, \sigma_{0}$ and $\sigma_{1}$ have to be estimated jointly, since we have no a priori value for them. We assume that the parameters are centered around the values given in Section 4.2, i.e.:

$$
\log E_{t h} \sim \mathcal{N}\left(\log \left(5.75 \times 10^{4}\right), 2\right), \log \sigma_{0} \sim \mathcal{N}(\log (0.065), 0.2), \log \sigma_{1} \sim \mathcal{N}(\log (0.1483), 0.5)
$$

The noise on the measurements is modeled by ${ }^{b} R^{-1 / 2}=0.2$, which corresponds to a noise of $25 \%$.

When all electrodiameters are considered together, the convergence of the estimation procedure is shown in Figure 9. As is the synthetic data case, the convergence of $\sigma_{0}$ is much slower than the other two parameters, which requires a large number of iterations.

For each individual electrode size, the convergence of the estimation procedure is shown in Figure 10. The estimated values differ significantly from one electrode diameter to the next, and $\sigma_{0}$ converges again slower than $E_{t h}$ and $\sigma_{1}$.

As we can see, the estimated parameters differ significantly between electrode diameters, especially for $0.3 \mathrm{~mm}$. We solve the direct problem with parameters estimated for each diameter individually ; and for the parameters estimated when considering all diameters simultaneously, see Figure 11. We can see the resulting distribution of $\sigma(\|\nabla u\|)$ for the three electrode diameters and for voltages of $200 \mathrm{~V}$ and $1000 \mathrm{~V}$. The distribution is very different, especially for $1000 \mathrm{~V}$. Table 6 presents the maximum relative error on the intensity, and shows that the best results are obtained when estimating the parameters only on the electrode of diameter $1.1 \mathrm{~mm}$. This is not 


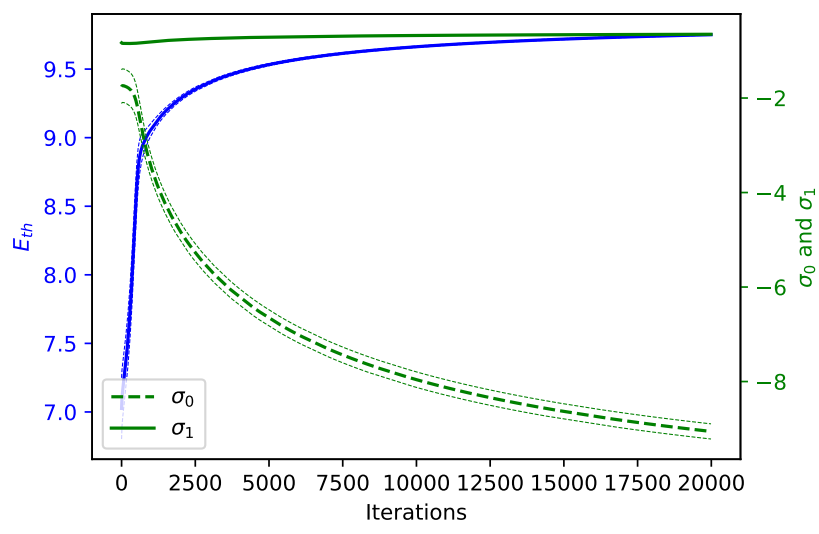

Figure 9. Estimation of $E_{t h}, \sigma_{0}$ and $\sigma_{1}$, after log-transformation. All voltages and all electrodes diameters are considered simultaneously. The thin dashed green and blue lines denote the standard deviation of the uncertainty on the estimation.
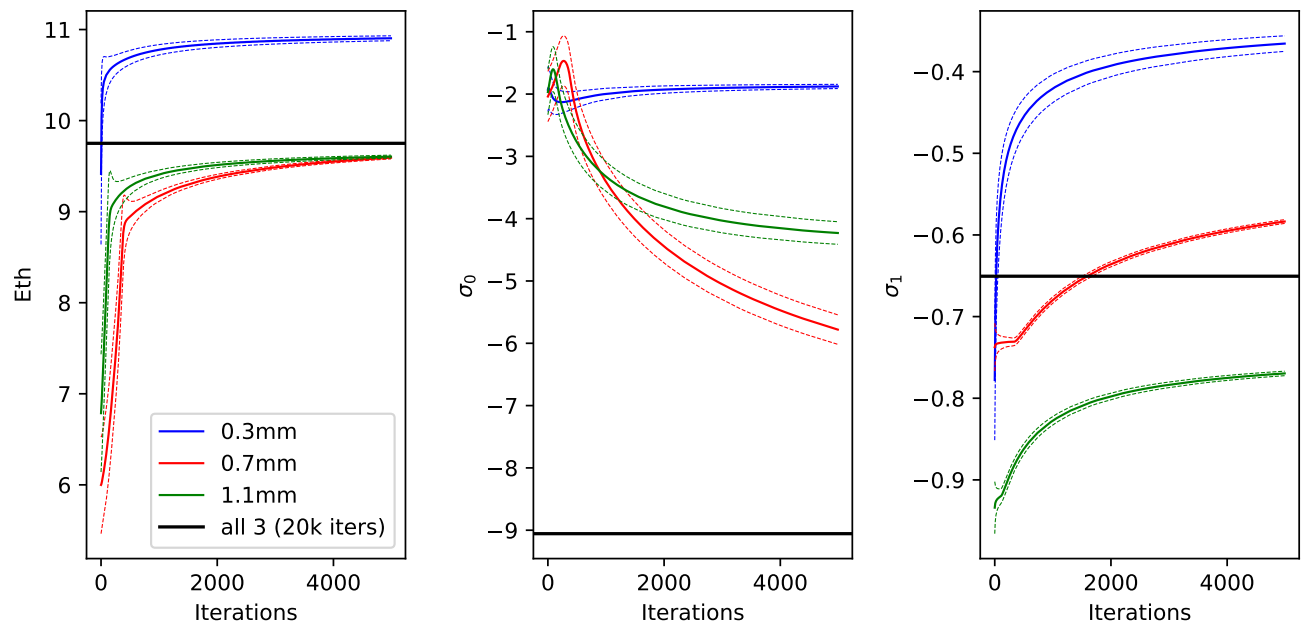

Figure 10. Estimation of $E_{t h}, \sigma_{0}$ and $\sigma_{1}$, after $\log$-transformation. All voltages are considered, but electrode diameters are considered separately. The thin lines denote the standard deviation of the uncertainty on the estimation.

surprising regarding to the relatively low values for the current for a diameter of $0.3 \mathrm{~mm}$, the quasi-equivalent values for diameters of 0.7 and $1.1 \mathrm{~mm}$ and also the relatively low values for low voltages (see Table 4 ).

Maybe, better results could be achieved by understanding better the impact on the measurement noise using for example synthetic data. One strategy could be for example to consider different values of the standard deviations of the observations in order to give less importance to the most noisy data. 

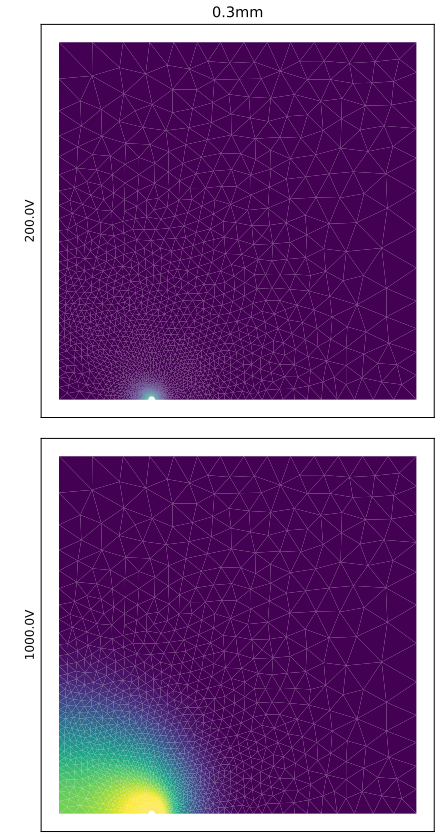

$0.3 \mathrm{~mm}$
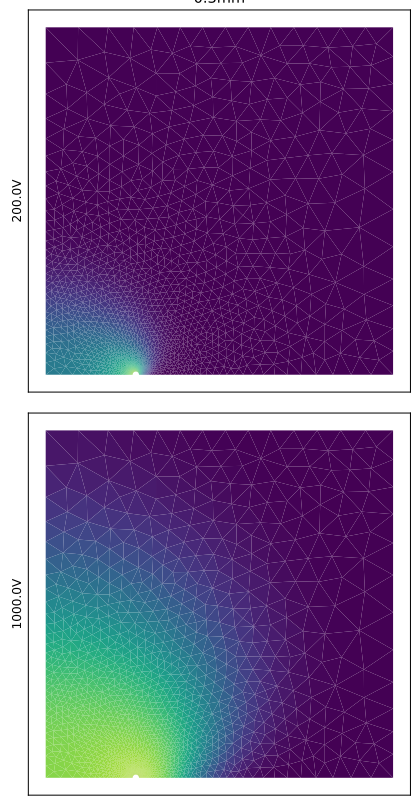

$0.7 \mathrm{~mm}$
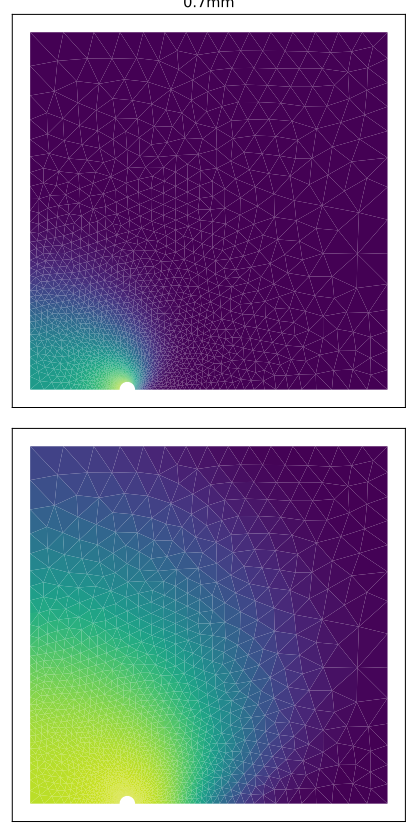

$0.7 \mathrm{~mm}$
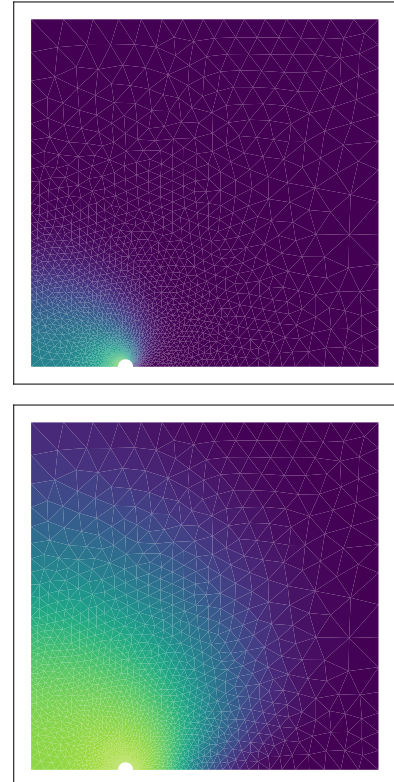

$1.1 \mathrm{~mm}$
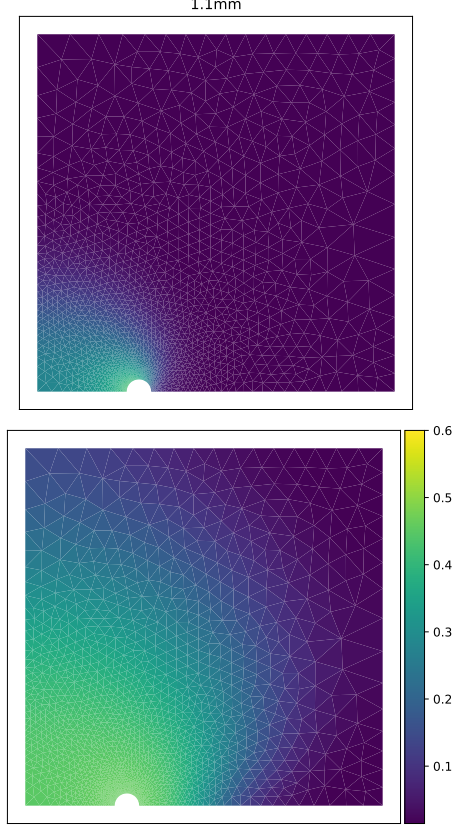

$1.1 \mathrm{~mm}$
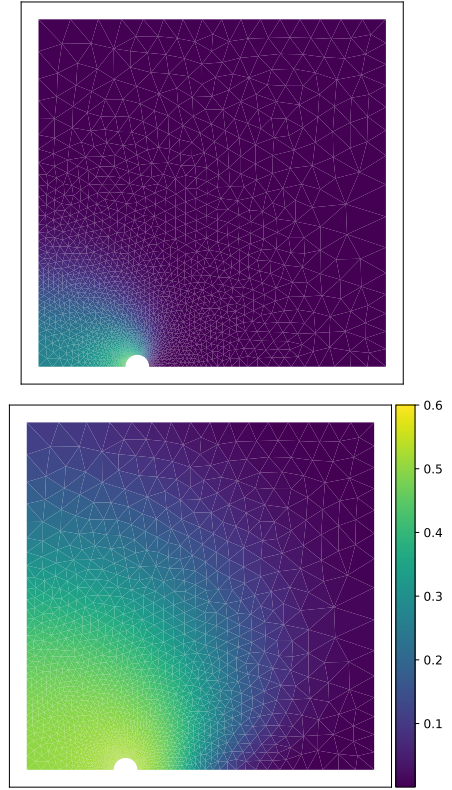

FiguRE 11. $\sigma(\|\nabla u\|)$ in $S / m$ for applied voltages $200 \mathrm{~V}$ and $1000 \mathrm{~V}$ and the three geometries, for the parameters estimated separately on each geometry (top 2 lines) and for the parameters estimated on all geometries simultaneously (bottom 2 lines). 


\begin{tabular}{cccc}
$\max _{V}\left|I_{\text {computed }}(V)-I_{\text {measured }}(V)\right| / I_{\text {measured }}(V)$ \\
\hline Geometry for estimation & \multicolumn{3}{c}{ Geometry for validation } \\
\hline & $0.3 \mathrm{~mm}$ & $0.7 \mathrm{~mm}$ & $1.1 \mathrm{~mm}$ \\
\cline { 2 - 4 } $0.3 \mathrm{~mm}$ & $3 \%$ & $33 \%$ & $26 \%$ \\
$0.7 \mathrm{~mm}$ & $52 \%$ & $24 \%$ & $31 \%$ \\
$1.1 \mathrm{~mm}$ & $27 \%$ & $24 \%$ & $15 \%$ \\
all & $31 \%$ & $34 \%$ & $26 \%$ \\
\hline
\end{tabular}

TABLE 6. Maximum relative error on the intensity across voltages, for each set of estimated parameters (one for each electrode diameter plus one for the estimated on all three simultaneously).

\section{Conclusion And Perspectives}

In this paper, we have compared different models of electroporation: the standard static model, the dynamical biphase model and the bidomain model in the same framework built using bioelectrical measurements on rabbit livers proposed by Sel et al. [19]. The solutions of the different models are realistic and can be qualitatively compared. Then we investigate a numerical calibration of the parameters only on the standard model. The strategy is based on a stochastic algorithm using an unscented transform for estimating parameters of static systems. The results on synthetic data are convincing. Finally, the estimation strategy is applied on the rabbit measurements and the attempt is promising even if the best fit is not achieved using all geometries simultaneously. As explained previously, this could be due to the measurements noise. This work allows to lay the ground of the calibration of electroporation models but many improvements have to be studied. The work on synthetic data has to be continued in order to better understand the estimation difficulties due to the measurements noise. Furthermore, the parameters estimation has to be applied on the bidomain model. Finally, concerning the dynamical biphase model, a dynamical version of the estimation strategy has to be written. The main difficulty lies in the fact that we need an efficient strategy which does not involve prohibitive computational times. To address this issue, Reduced-order Kalman filter method, introduced by Moireau and Chapelle [18], will be investigated in a near future.

\section{ACKNOWLEDGEMENTS}

The authors would like to thank very warmly Philippe Moireau and Sébastien Impériale from the Inria team MEDISIM and Olivier Gallinato from the SophiaGenetics company for their help and their fruitful discussions. This research was granted by the plan Cancer NUMEP, PC201615, led by C. Poignard.

\section{REFERENCES}

[1] M. S. Alnæs, J. Blechta, J. Hake, A. Johansson, B. Kehlet, A. Logg, C. Richardson, J. Ring, M. E. Rognes, and G. N. Wells. The fenics project version 1.5. Archive of Numerical Software, 3(100):9-23, 2015.

[2] M. Breton, F. Buret, L. Krähenbühl, M. Leguèbe, L. M. Mir, R. Perrussel, C. Poignard, R. Scorretti, and D. Voyer. NonLinear Steady-State Electrical Current Modeling for the Electropermeabilization of Biological Tissue. IEEE Transactions on Magnetics, 51(3):1-4, Mar. 2015.

[3] P. Colli Franzone and G. Savaré. Degenerate evolution systems modeling the cardiac electric field at micro and macroscopic level. Progress in Nonlinear Differential Equations and Their Applications, 50:49-78, 2002.

[4] A. Collin and S. Imperiale. Mathematical analysis and 2-scale convergence of a heterogeneous microscopic bidomain model. Mathematical Models and Methods in Applied Sciences, 28(05):979-1035, 2018.

[5] R. V. Davalos, L. M. Mir, and B. Rubinsky. Tissue Ablation with Irreversible Electroporation. Annals of Biomedical Engineering, 33(2):223-231, Feb. 2005. 
[6] R. V. Davalos, B. Rubinsky, and L. M. Mir. Theoretical analysis of the thermal effects during in vivo tissue electroporation. Bioelectrochemistry, 61(1):99-107, Oct. 2003.

[7] M. Deville. Mathematical Modeling of enhanced drug delivery by mean of Electroporation or Enzymatic treatment. PhD Thesis, Université de Bordeaux, 2017.

[8] J. Edd, L. Horowitz, R. Davalos, L. Mir, and B. Rubinsky. In Vivo Results of a New Focal Tissue Ablation Technique: Irreversible Electroporation. IEEE Transactions on Biomedical Engineering, 53, 2006.

[9] J. Gehl. Investigational treatment of cancer using electrochemotherapy, electrochemoimmunotherapy and electro-gene transfer. Ugeskr Laeger., 165, 2005.

[10] J. Gehl, T. Skovsgaard, and L. M. Mir. Vascular reactions to in vivo electroporation: characterization and consequences for drug and gene delivery. Biochimica et Biophysica Acta (BBA) - General Subjects, 1569(1):51 - 58, 2002.

[11] A. Golberg and B. Rubinsky. A statistical model for multidimensional irreversible electroporation cell death in tissue. Biomedical Engineering Online, 9(13), 2010.

[12] A. Ivorra, J. Villemejane, and L. M Mir. Electrical modeling of the influence of medium conductivity on electroporation. Physical chemistry chemical physics : PCCP, 12:10055-64, 2010.

[13] S. J. Julier. The spherical simplex unscented transformation. In Proceedings of the 2003 American Control Conference, 2003., volume 3, pages 2430-2434. IEEE, 2003.

[14] S. J. Julier and J. K. Uhlmann. Unscented filtering and nonlinear estimation. Proceedings of the IEEE, 92(3):401-422, 2004.

[15] M. Leguèbe, M. Notarangelo, M. Twarogowska, R. Natalini, and C. Poignard. Mathematical model for transport of DNA plasmids from the external medium up to the nucleus by electroporation. Mathematical Bioscience journal, 2017.

[16] M. Leguèbe, A. Silve, L. M. Mir, and C. Poignard. Conducting and permeable states of cell membrane submitted to high voltage pulses: Mathematical and numerical studies validated by the experiments. Journal of Theoretical Biology, 360:83-94, Nov. 2014.

[17] L. M. Mir, S. Orlowski, J. Belehradek, and C. Paoletti. Electrochemotherapy potentiation of antitumour effect of bleomycin by local electric pulses. European Journal of Cancer and Clinical Oncology, 27(1):68 - 72, 1991.

[18] P. Moireau and D. Chapelle. Reduced-order unscented Kalman filtering with application to parameter identification in largedimensional systems. ESAIM: Control, Optimisation and Calculus of Variations, 17(2):380-405, 2011.

[19] D. Sel, D. Cukjati, D. Batiuskaite, T. Slivnik, L. M. Mir, and D. Miklavcic. Sequential finite element model of tissue electropermeabilization. IEEE Transactions on Biomedical Engineering, 52(5):816-827, 2005.

[20] D. Voyer, A. Silve, L. M. Mir, R. Scorretti, and C. Poignard. Dynamical modeling of tissue electroporation. Bioelectrochemistry, 119:98 - 110, 2018. 